\title{
Evaluación comparativa de productos, servicios y procesos en modalidad virtual que ofrecen las instituciones de educación superior en Colombia
}

\author{
Marcela Cardona, Compensar-Unipanamericana, Colombia
}

\begin{abstract}
Resumen: La educación virtual en Colombia, ha tenido un notable crecimiento en los últimos años. El gobierno nacional ha promovido políticas que permiten ampliar la cobertura y formar a la población principalmente en carreras técnicas y tecnológicas que den respuesta a las necesidades del mercado laboral. Son numerosas las razones por las cuales las personas no acceden a programas de formación superior, entre ellas factores de tipo económico, carencia de tiempos para asistir a un aula regular, ubicación en regiones apartadas del país entre otros.A través del Ministerio de Educación MEN y de las TIC (Tecnologías de la Información y la Comunicación) la educación virtual en Colombia ha presentado una constante evolución. Esto ha permitido que en un ejercicio armónico entre pedagogía y tecnología, se creen espacios propicios de formación académica en nivel superior. Los diferentes entes gubernamentales vieron la necesidad de diferentes instituciones y personas de acceder a una educación de calidad que cumpliera con los parámetros establecidos de acuerdo a sus necesidades y es así como a través de la educación virtual se lograr solventar esta necesidad y proporcionarle a los diferentes actores una herramienta útil que les suministre una educación a la altura que por derechos les corresponde. Es así como a través de diferentes entes gubernamentales se logra la cobertura de gran parte de la población para acceder a educación virtual a través del otorgamiento de subsidios para crear sitios especializados para el acceso a internet y programas especificos. Este artículo presenta una evaluación comparativa de productos, servicios y procesos en modalidad virtual que ofrecen las instituciones de educación superior en Colombia a nivel de pregrado. El proceso de análisis de los resultados se realiza presentando la evaluación desde tres aspectos: 1) Generalidades de los programas virtuales 2) Servicios ofrecidos por las instituciones de educación superior en modalidad virtual 3) Presencia en internet y redes sociales como apoyo a la comunicación efectiva.
\end{abstract}

Palabras claves: educación virtual, servicios de formación virtual, procesos de formación virtual, redes sociales

\begin{abstract}
Virtual education in Colombia, has had a remarkable growth in recent years. The national government has promoted policies that expand coverage and train the population mainly in technical and technological fields that respond to the needs of the labor market. There are numerous reasons why people do not have access to higher education programs, including economic factors, lack of time to attend a regular classroom, located in remote regions of the country, among others. Through the Ministry of Education MEN and ICT (Information and Communication Technology) virtual education in Colombia has provided a constant evolution. This has allowed that in a harmonious connection between pedagogy and technology, exists the creation of appropriate areas in higher level education. This paper presents a comparative evaluation of products, services and procedures in virtual mode offered by institutions of higher education in Colombia at the undergraduate level. The process of analysis of the results focuses on three aspects: 1) Overview of virtual training programs 2) Services offered in virtual modality by institutions of higher education 3) Presence on the Internet and social networks to support effective communication.
\end{abstract}

Keywords: Virtual Education, Virtual Training Services, Social Networking

\section{Introducción}

$\mathrm{H}$ ace menos de dos décadas la educación en Colombia era un privilegio para pocos, la oferta académica era reducida y se debía contar con una cantidad de dinero significativa para poder financiarla. Con el fin de solucionar estos inconvenientes y teniendo en cuenta que iba creciendo la demanda de personas que querían ingresar a la educación superior, diversas entidades le dieron a muchos estudiantes potenciales la posibilidad de adquirir un crédito de financiamiento para su carrera.

Revista Internacional de Tecnologías en la Educación

Volumen 2, Número 1, 2015, <http://sobrelaeducacion.com>, ISSN 2386-8384

(C) Global Knowledge Academics. Marcela Cardona. Todos los derechos

reservados. Permisos: soporte@gkacademics.com 
Con lo anterior se les brindó la oportunidad a diferentes estudiantes a acceder a programas en los diferentes ciclos técnico, tecnológico, profesional y con ello tener la posibilidad de acceder a cargos administrativos de carácter empresarial.

Estas oportunidades les brindan a personas que no tienen el tiempo suficiente para ir a un aula de clase debido a múltiples ocupaciones, estudiar como cualquier otra persona y convertirse en un profesional que tiene mayores oportunidades en el campo profesional y las demandas del mercado.

Las instituciones de educación superior buscan brindar al estudiante espacios dinámicos, servicios que sean de su interés y que brinden una solución rápida y oportuna en relación con sus necesidades, según Murcia (2004) "en materia de educación virtual, es preciso reconocer y comprender este dinámico, histórico, y cada vez más significativo proceso de convergencia" (p.100), la posibilidad de crear espacios para compartir en tiempo real y en cualquier momento hace que una solución sea la generación de espacios en modalidad virtual mediante programas de formación que se enseñan a través de la web, esto sin necesidad de tener que acercarse a un aula de clase. Sin embargo ¿Cuáles son los productos, servicios y procesos en modalidad virtual ofrecidos por las instituciones de educación superior en Colombia?, ¿es posible a través de una evaluación de los mismos, identificar buenas prácticas con el fin de ser trasferidas a los procesos adelantados por las instituciones de educación superior que ofrecen servicios en esta modalidad?

A través de las preguntas enunciadas anteriormente, se busca alcanzar una visión general de la educación virtual en Colombia, evaluar los productos, servicios y procesos de formación virtual a nivel de pregrado, con el fin de transferir el conocimiento de las mejores prácticas y su aplicación a círculo de negocios.

Para ello se han establecido como objetivos específicos, en primer lugar, indagar los hechos más representativos de la educación virtual en Colombia entre 2010-2012, y en segundo lugar comparar los servicios de comunicación, apoyo al estudiante y ventajas competitivas ofrecidas por las instituciones de educación superior en Colombia frente a la modalidad virtual.

Los factores más destacados que han provocado el nacimiento y posterior desarrollo de la educación a distancia en Colombia, han sido los avances sociopolíticos, la necesidad de aprender a lo largo de la vida, la escasez de los sistemas convencionales, los avances en el ámbito de la ciencia y la educación enfocada a las transformaciones tecnológicas. (UNESCO, 2003).

La educación a distancia ha obtenido significativos cambios a través del tiempo, los cuales se pueden definir en tres pasos importantes: la correspondencia, telecomunicaciones y telemática. En Colombia nace un modelo latinoamericano con las llamadas escuelas radiofónicas, iniciadas por acción cultural popular en 1947 a partir de este momento se establecieron programas similares en otros países.

Es por esto que diferentes instituciones en Colombia identificaban y analizaban la educación virtual como un camino a seguir frente a los diferentes inconvenientes que se pudieran relacionar con las exigencias del mercado actual, esto debido al poco tiempo con el que se cuenta frente a desplazamientos, el manejo de una vida personal y laboral activa en la cual el tiempo se convierte en factor determinante a la hora de realizar estudios.

A partir de lo anterior se busca generar estrategias para lograr que más personas puedan acceder a educación superior y tener mayores oportunidades laborales.

En los últimos años la oferta de programas virtuales ha tenido un crecimiento alto, según Montes (2012) "la cobertura de Internet en el país todavía es limitada. A futuro se espera que con el Plan Nacional de Fibra Óptica esta situación mejore considerablemente", las alternativas de financiación han ido aumentando con el fin de que la población colombiana tenga más oportunidades frente a su proceso de formación, es de anotar que la educación en Colombia se concentra en un porcentaje considerable en las principales ciudades, partiendo de que con la modalidad virtual el estudiante puede estar ubicado en cualquier lugar del país.

A partir del 2015 el gobierno tiene proyectado alcanzar la cobertura de más del 98\% de la población, para esto ha realizado diferentes inversiones con el fin de cubrir una parte considerable de la población para que accedan a internet. El siguiente paso es obtener los equipos necesarios para que se puedan aprovechar al máximo estas tecnologías. 
El progreso de la educación virtual ha sido significativo en diferentes aspectos como lo son las tendencias y las herramientas que se utilizan para poder brindar esta modalidad al público.

Actualmente existen treinta y tres instituciones de educación superior que cuentan con registro calificado por el Ministerio de educación en Colombia, estas instituciones creen en la modalidad virtual y han ampliado sus portafolios con carreras de pregrado y postgrado teniendo en cuenta la demanda actual.

Estás instituciones brindan a los estudiantes en modalidad virtual servicios como soporte técnico, asesoría en psicología, en las actividades de bienestar cuentan con actividades extracurriculares que ofrezcan un apoyo a los estudiantes. La idea es que a través de estos beneficios para su educación, el estudiante se sienta igual de importante que otro estudiante que tiene la posibilidad de estudiar de manera presencial.

Al tener acceso a los procesos institucionales de un estudiante presencial, el estudiante de modalidad virtual podrá hacer uso de ellos en cualquier momento de su carrera y sentirá un respaldo de la institución, que aunque no lo ve siempre en sus instalaciones si hace uso de la plataforma y merece las ventajas a las que tienen acceso estudiantes presenciales.

\section{Método}

Enmarcados en la investigación de mercados que se define "como un enfoque sistemático y objetivo orientado al desarrollo y provisión de información aplicable al proceso de toma de decisiones" (Tamayo, 1999, p.52), se involucran varios participantes entre ellos clientes, consumidores y público en general mediante información que permite identificar y definir oportunidades teniendo en cuenta la pregunta problema, en esta etapa de la investigación la población está enfocada en las instituciones Colombianas que ofrecen modalidad virtual en sus portafolios, Nareska (2004) afirma que "la investigación de mercados comprende la identificación, acopio, análisis, difusión y aprovechamiento de la información" (p.7)

Mediante un proceso de Bechmarketing comparativo se busca recolectar información de las diferentes instituciones de educación superior que ofrecen modalidad virtual en Colombia "el Bechmarketing competitivo permite abrirse a las ideas del exterior, y compararse con otras empresas para aprender" (Associaciód'Empreses i Institucions 22@Barcelona, 2011, p.25) con este análisis se busca identificar variables y servicios relevantes en modalidad virtual con el fin de ser estudiados teniendo en cuenta lo que se ofrece actualmente en el mercado Colombiano de esta forma se logra un estudio de mercado efectivo en relación con las variables identificadas.

Fernández (2004) afirma que el muestreo probabilístico "permite evaluar y controlar las desviaciones en las estimaciones de las características objeto de estudio" (p.154). La muestra fue tomada de las instituciones avaladas por el Ministerio de Educación en Colombia mediante las estadísticas de SNIES, para un total del tamaño del universo de 33 instituciones, con una probabilidad de ocurrencia del 0.5 , tenemos un nivel de confiabilidad del $99 \%$ y un error máximo de estimación del 1\% con una elección total de muestra de 33 instituciones.

Como instrumento de recolección de información se hace uso de la lista de cotejo, la cual "es una presentación ordenada de acciones o características deseadas o no en la actuación que debe observarse" (D“Agostino, 2007, p.32). La lista se ha estructurado a partir de los siguientes ítems: 1) Generalidades de los programas virtuales 2) Servicios ofrecidos por las instituciones de educación superior en modalidad virtual 3) Presencia en internet y redes sociales como apoyo a la comunicación efectiva. La información se recolecta a partir de lo publicado por cada una de las instituciones en sus portales de Internet apoyado por la solicitud a través del correo electrónico para identificar tiempos de respuesta y procedimientos de solicitud de información por parte de los aspirantes.

Teniendo en cuenta la información obtenida por el Sistema Nacional de Información de la Educación Superior (SNIES) donde se encuentran registradas 33 instituciones con registro calificado en sus programas y 2 programas avalados con registro de alta calidad: La Fundación UniversitariaCEIPA y la Universidad EAN con el programa Administración de Empresas. 
Tabla 1: Instituciones analizadas

\begin{tabular}{|l|}
\hline \multicolumn{1}{|c|}{ Instituciones de Educación Superior } \\
\hline Corporación Universidad de la Costa CUC \\
\hline Corporación Universitaria Minuto de Dios -UniMinuto- \\
\hline Fundación de Estudios Superiores Comfanorte -F.E.S.C.- \\
\hline Fundación Escuela Colombiana de Mercadotecnia -eSCOLME- \\
\hline Fundación Universitaria Católica del Norte \\
\hline Fundación Universitaria de San Gil - UniSanGil - \\
\hline Fundación Universitaria Esumer \\
\hline Fundación Universitaria los Libertadores \\
\hline Fundación Universitaria Maria Cano \\
\hline Fundación Universitaria-Ceipa- \\
\hline Politécnico Grancolombiano \\
\hline Pontificia Universidad Javeriana \\
\hline Tecnológica FITEC \\
\hline Unipanamericana - Fundación Universitaria Panamericana \\
\hline Universidad Antonio Nariño \\
\hline Universidad Autónoma de Bucaramanga-UNAB- \\
\hline Universidad Autónoma del Caribe \\
\hline Universidad Católica de Pereira \\
\hline Universidad de Antioquia \\
\hline Universidad de Medellín \\
\hline Universidad de Nariño \\
\hline Universidad de Pamplona \\
\hline Universidad de San Buenaventura \\
\hline Universidad del Quindío \\
\hline Universidad del Tolima \\
\hline Universidad EAN \\
\hline Universidad Industrial de Santander \\
\hline Universidad Manuela Beltran-UMB- \\
\hline Universidad Militar-Nueva Granada \\
\hline Universidad Nacional Abierta y a Distancia UNAd \\
\hline Universidad Santiago de Cali \\
\hline Universidad Tecnológica de Bolivar \\
\hline
\end{tabular}

Fuente: Elaboración propia, 2014.

\section{Resultados y discusión}

\section{Generalidades de los programas virtuales}

En Colombia las instituciones de educación virtual han ingresado a esta modalidad porque la ven como una alternativa para brindar la oportunidad de acceso a la educación superior y ampliación de la cobertura, así como una alternativa para reducir la brecha social. La formación educativa a través de e-learning se caracteriza por la flexibilidad en tiempo y espacio, dentro de sus objetivos principales encontramos el desarrollo de conocimientos, habilidades y aptitudes necesarias para enfrentar el mercado laboral e impactar como profesionales el contexto.

La educación virtual en Colombia cada vez tiene mayor cobertura, se puede destacar que la ciudad del país donde se ofrece más esta modalidad es en Bogotá con un $36 \%$, seguida de Medellín $18 \%$ y Bucaramanga $9 \%$ ya que han sido ciudades donde ha sido posible el crecimiento de las nuevas tecnologías y la generación de estrategias por instituciones de educación superior en incursionar en esta modalidad. En Colombia las instituciones utilizan diferentes tecnologías para ofertar cursos en línea, crear comunidades, acceder a bibliotecas y aulas virtuales. Además, las instituciones plantean diferentes formas para establecer una buena comunicación entre el alumno y el docente pues de 
esta manera es más sólida y permanente la enseñanza. De acuerdo con el DANE (2002). Las instituciones de educación superior que ofertan programas en modalidad virtual en su mayoría tienen un $97 \%$ con programas de pregrado, un $58 \%$ cuenta adicionalmente con programas de postgrados, y una minoría cuenta con cursos de extensión.

Según Cortes, Parra \& Cardona (2012) "Los docentes han identificado que la creación de material digital permite que los estudiantes participen en la generación de estos contenidos y sean actores activos del proceso de aprendizaje" ( $\mathrm{p}, 3)$, con respecto a la incorporación de las TIC al espacio de clase estas se caracterizan por ser practicas lúdicas lo cual conlleva a que los contenidos sean más llamativos para el estudiante generando motivación en el mismo a través de una nueva manera de hacer las cosas fomentando mayor retentiva frente a la información; de igual manera las TIC le permiten al docente enriquecer los procesos didácticas, mostrándole al estudiante diversos estilos de aprendizaje bajo una relación interactiva

Haciendo énfasis en la incorporación de las TIC en el aula de clase, Vallejo (2011) afirma:

En la incorporación de la Tics al aula de clase estas permiten al docente dimensionar su utilidad en su proceso de enseñanza enriqueciendo sus procesos didácticos y facilitando el impacto hacia él estudio a través de diversos estilos de aprendizaje para que haya mayor motivación. (p.6).

Las TIC han generado una serie de recursos y opciones para que el estudiante comprenda de manera más fácil y tenga un proceso que no sea monótono entre estos encontramos: Dentro del primer recurso para analizar y presentar la información se encuentra Prezi, caracterizado por ser una herramienta que muestra de manera precisa la información, por el lado de encontrar actividades prediseñadas encontramos Eduteka el cual es un portal gratuito que pone a disposición de los estudiantes, centenares de contenidos formativos e informativos que les ayudan a enriquecer, con el uso de las TIC, sus ambientes escolares de aprendizaje, además, diseña e implementa espacios interactivos en línea con recursos útiles y valiosos para los docentes; con respecto a los recursos para hacer evaluaciones encontramos los formularios de Google Docs por medio de los cuales el docente genera la evaluación, enviándosela al estudiante y este genera él envío de la misma de forma inmediata al realizarla un ejemplo de lo que actualmente se realiza en la Fundación Universitaria Panamericana, con el fin de contar con evidencias claras del proceso en relación con la auto y coevaluación de la misma, por último encontramos los recursos para generar material audiovisual dentro de ellos encontramos dentro de los más reconocidos Audacity un programa para la generación propia de audios y Moviemaker en el cual se crean videos de diferentes maneras

En relación con la metodología de los ciclos propedéuticos "los cuales se concibe como un conjunto de competencias relacionadas con el conocimiento, su uso y aplicación en diferentes contextos, así como el desarrollo de actitudes, responsabilidades y valores" (CONACES), estos brindan la posibilidad a los estudiantes de ser profesionales en tres niveles que se conforman entre sí, permitiendo que puedan avanzar en su proceso de formación profesional. Es por esto que el $61 \%$ de las instituciones cuenta con este sistema para ampliar la posibilidad de mejorar los modelos de educación, de esta forma se genera una retroalimentación continua que apoye el fortalecimiento de la educación.

Teniendo en cuenta la organización administrativa de los grupos en el proceso de formación se consideran aspectos como: número de estudiantes por grupo, en la investigación se puedo destacar que el $24 \%$ de instituciones conforman sus grupos de aproximadamente 20 a 30 estudiantes, seguido de un $21 \%$ de instituciones que informan conformar grupos entre 10 y 20 estudiantes ya que informan realizar un proceso personalizado y donde los tutores y los estudiantes puedan tener una mayor interacción y comunicación, lo cual puede permitir mejores resultados en el aprendizaje, debemos tener en cuenta que esto también puede estar relacionado con la oferta académica y la población que se inscribe en esta modalidad, por último es importante destacar que un $48 \%$ de las instituciones no brindaron información sobre este aspecto

Los convenios interinstitucionales permiten establecer relaciones para obtener ayudas que promuevan el intercambio académico y promueven la movilidad estudiantil.. Según las estadísticas, un $30 \%$ de instituciones cuentan con convenios internacionales que facilitan la interacción cultural y el 
intercambio, el 36\% cuentan con alianzas empresariales que permiten y brindan a los estudiantes la facilidad de empezar sus estudios de formación profesional ya que ellos pueden obtener algunos descuentos, el 39\% genera convenios a nivel financiero para brindar un mayor portafolio a sus estudiantes en este aspecto, es decir que más de un $67 \%$ de las instituciones cuentan con convenios con otras instituciones para generar mayores conocimientos y credibilidad.

Carreras como Administración de Empresas o Contaduría, en la actualidad mantienen un alto grado de demanda, es por esta razón que la oferta más amplia es la de ciencias empresariales, la ingeniería se mantiene como una de las facultades fuertes de las universidades, el campo de la comunicación queda un poco relegado, y las ofertas para carreras de educación y licenciatura van en crecimiento. Podemos identificar que en las instituciones educativas de nuestro país sigue dominando ampliamente el periodo semestral en el desarrollo de las carreras y apenas el periodo trimestral se maneja en un reducido grupo de instituciones. Con respecto a los créditos, en los programas técnicos estos oscilan entre (50-79) créditos, los créditos de los programas tecnológicos se encuentran entre (80-139) y en programas profesionales los créditos están entre (139-160).

\section{Servicios ofrecidos por las instituciones de educación superior en modalidad virtual}

Las instituciones evaluadas en su mayoría cuentan con servicios de bienestar de manera virtual, estas actividades son en menor proporción comparadas con la que se efectúan a nivel presencial, el ideal de estas actividades es a través de la interacción virtual brindarle una acompañamiento constate al estudiante promoviendo acciones que mejoren sus estados de ánimo, como es el caso del apoyo en psicología en línea una actividad por medio de la cual se le da un apoyo a la persona para que esté tranquila o solucione algún problema. La mayoría de las universidades también cuenta con actividades culturales que conlleva a que el estudiante haga uso de actividades dinámicas. Por otro lado los programas radiales y de televisión también son de gran importancia en la mayoría de instituciones ya que a través de esto dan a conocer información relevante. Algunas de las instituciones no cuentan con el servicio de bienestar universitario, y pocas cuentan con centros virtuales para los egresados, centros virtuales para los discapacitados, ya que lo ven como una minoría.

El área de soporte técnico es de gran importancia para todas las universidades del país ya que brindan un conjunto de herramientas y nuevas tecnologías para dar soluciones rápidas y efectivas a los problemas que se puedan presentar, el total de las instituciones cuenta con correos electrónicos para dar respuesta a los interrogantes de los estudiantes. Igualmente, hacen uso de la línea de atención y los manuales en líneas en formatos PDF, que facilitan la información.

La interacción a través de una plataforma virtual es importante para el desarrollo de espacios de aprendizaje idóneos donde la institución pueda tener un lugar personalizado con sus estudiantes, es por esto que $73 \%$ de las universidades cuentan con plataformas como Moodle y el $27 \%$ con Blackboard ya que ayudan a los docentes a crear ambientes de estudio y así generar mayor conocimiento basándose en las habilidades y conocimientos. La creación de estos espacios formales genera un seguimiento a los accesos de los estudiantes, fechas de interacción, publicación de evidencias, trabajo colaborativo y de debate en foros y audio foros con la posibilidad de ser evaluados directamente, manejo de un centro de calificaciones, uso de bases de datos entre otros.

La tendencia indica que el lapso en el que la mayoría de instituciones dan respuesta a los correos es de un día, lo cual quiere decir que el correo sigue siendo un medio efectivo para solicitar y recibir información, sus tiempos de respuesta cortos, permiten el buen manejo de la información y la efectividad del medio. Se evidencia que la herramienta del chat no es muy utilizada en las páginas de las universidades, sin embargo se encuentran casos donde la herramienta no funcionaba o el tiempo de respuesta era muy largo, es decir las pocas instituciones que lo utilizan al parecer no le prestan mucha atención a un medio que puede ser más práctico y eficaz para conseguir información que el mismo correo, esto debido a la inmediatez de los mensajes y el tiempo de respuesta por parte de los receptores. El teléfono es un medio que prevalece, por ser el más inmediato para conseguir información, y a pesar de que ya existen otras herramientas gracias al internet, las líneas telefónicas siguen siendo más efectivas en este caso. 
En relación con las bases de datos estás permiten almacenar y recopilar información, cada vez más existen diferentes alternativas ya que son herramientas que facilitan la investigación y la labor académica. La más relevante es EBSCO porque permite obtener datos en documentos completos y realiza publicaciones de manera periódica en diferentes áreas, al igual que Legiscomex y E-libro pero tienen menos participación en las instituciones analizadas. Las que tiene menos participación en las universidades que hacen uso continuo de las bases de datos son Bibliotechnia, Noticier Oficial, Pro Quest.

\section{Presencia en internet y redes sociales como apoyo a la comunicación efectiva}

La presencia de internet y las redes sociales en la vida de las personas ha crecido en los últimos años, "el mundo educativo no puede permanecer ajeno ante fenómenos sociales como este que está cambiando la forma de comunicación entre las personas." (De Haro, J, 2010) la educación busca formar a las personas teniendo en cuenta las tendencias actuales y las tendencias a futuro siempre visualizando diez años con el fin de poder identificar a futuro las nuevas estrategias o mecanismos que se puedan utilizar.

Estas redes se han convertido en un gran apoyo para los docentes, que a través de cada una de las páginas sociales motivan la participación de los estudiantes en sus clases. Además, las clases se convierten en un estímulo ya que el cerebro las considera como páginas de ocio y el estudiante ingresa a ellas por su propio gusto.

A través de la interacción que se evidencia en estas redes, el estudiante y el docente tienen un vínculo más cercano. Además, como las redes son permiten que cada contenido se suba rápidamente, la interacción y el acceso a información es inmediato.

En la modalidad virtual, la presencia de la institución es importante no solo a nivel de la página principal, ya que debe estar presente en las principales redes sociales puesto que muchos estudiantes solicitan o colocan información relevante y de utilidad; la comunicación vía internet es un canal muy eficaz entre la institución y el estudiante. En la investigación se pudo observar que la red social más utilizada es Facebook, ya que esta permite colocar contenido multimedia para que el mensaje llegue de una manera más atractiva, por otro lado Twitter brinda el espacio para opinar sobre determinadas temáticas, y a través de YouTube se muestran videos que tengan que ver con las actividades realizadas dentro de la institución, es de anotar que las instituciones también tienen presencia en otras redes sociales como Flickr y Google +.

Facebook permite subir cualquier tipo de información o material de apoyo si es necesario, a través de esta red se generan comunidades o grupos relacionados con la institución, sus facultades, bienestar entre otros, las instituciones investigadas tienen presencias diaria o permanente en Facebook, y suben contenido e interactúan con los estudiantes.

Facebook es la red social que cuenta con más usuarios en comparación con las demás redes sociales, ya que se caracteriza por generar aplicaciones interactivas. Por ende son más llamativas para los estudiantes, a nivel general la mayoría de personas cuenta con un usuario en Facebook con uso constante, en el caso de las instituciones evaluadas la mayoría de estas tiene un número superior a 2000 seguidores, estas personas de igual manera motivan al crecimiento y participación de más personas que se puedan interesar por la institución. Se pueden crear Fan Page y Grupos para la interacción entre personas del mismo segmento.

Twitter es un micro blog que caracteriza por generar contenido en tan solo 140 caracteres, enlazando también documentos, archivo multimedia entre otros. Además, es una herramienta de comunicación que está en constante crecimiento ya que los estudiantes la tienen instalada en sus dispositivos móviles, por ende pueden tener un acercamiento más con la universidad. De las instituciones evaluadas el $93 \%$ de estas cuentan con un usuario en esta red, el $7 \%$ no tienen presencia por este medio, aquellas que si tienen presencia en su mayoría generan contenido esporádicamente cada tres o dos días.

A diferencia de las redes mencionadas anteriormente YouTube no es una red social utilizada de manera constante por las instituciones. A través de esta se dan a conocer principalmente videos relacionados con aspectos de las universidades, actividades, en algunos casos críticas a las institu- 
ciones o un paseo a través del campus de las mismas. La publicación en esta red social es esporádica, su uso se ve reflejado entre cuatro a ocho días, y la cantidad de comentarios de los videos no es alta, por otro lado un porcentaje significativo de las instituciones no posee usuario en esta red social.

Dependiendo del contenido generado por la institución a través de Twitter esta no solo contará con seguidores que sean allegados a la institución también tendrá interacción con personas del común que se interesen por sus publicaciones. Teniendo en cuenta las instituciones evaluadas dos de ellas no tiene presencia en esta red, 6 de ellas tiene entre 500 y 1000 seguidores un número significativo ya que el contenido no es constante, por otro lado 19 de las instituciones cuenta con un número superior a 2000 seguidores y más de 1.500 tweets. Es importante aprovechar el uso de esta red social ya que a través de esta la universidad se da cuenta de cuáles son los temas que marcan tendencia en la red y pueden hacer uso de esto para ganar mayores seguidores y popularidad.

Podemos decir que "las comunidades virtuales de aprendizaje se evidencian como una red social y las redes a su vez como mediadoras en los procesos de aprendizaje, las cuales a través de estrategias de comunicación efectiva y orientaciones pedagógicas pertinentes" (Pineda, E. Telléz, F. \& Meneses, 2013) es por esto que las redes sociales pueden ser un apoyo para el desarrollo de espacios de discusión entre los estudiantes y un mecanismo de apoyo en el desarrollo de estrategias de debate y colaboración.

\section{Conclusiones}

- Para consolidar el Bechmarketing se realizó una investigación a 33 instituciones educativas de Colombia, solicitando a estas información sobre los programas que ofertaban en modalidad virtual, y como era el proceso de admisión, algunas respondieron la solicitud de forma inmediata, otras se demoraron en dar respuesta entre uno o dos días, y a la fecha algunas instituciones no han respondido a la solicitud.

- Las instituciones de educación superior en Colombia que ofrecen modalidad virtual a nivel de pregrado en su mayoría son del sector privado.

- El costo de los programas ofertados por las instituciones en Colombia en la modalidad virtual depende en gran parte de la universidad y su reconocimiento; algunas ofrecen programas similares, otras se caracterizan por tener costos más elevados con respecto a otras que tienen precios que se acomodan a la necesidad de las personas interesadas en adquirir estudios profesionales y cuyos recursos económicos no son tan altos.

- La oferta de programas virtuales se encuentra principalmente en programas de pregrado, dentro de estos se encuentran las carreras técnicas y tecnológicas, en menor cantidad se ofertan programa de postgrados, en esta categoría se encuentran las maestrías y especializaciones.

- El soporte técnico de las universidades en Colombia en su totalidad cuentan con línea de atención telefónica para atender a los usuarios, algunas cuentan con chat en línea pero no se encuentra fácilmente en la página web o no está funcionando, otras cuentan con manuales en pdf, archivos de audio o video para familiarizar a los estudiantes que están interesados en hacer parte de ellas.

- Las instituciones generan convenios o alianzas con entidades financieras o empresariales tanto a nivel nacional como internacional para facilitar el acceso a una educación superior al estudiante y para promover intercambios de proyectos.

- La presencia en internet para la las instituciones es importante ya que de esta manera se dan a conocer de forma más efectiva a todas las personas que hacen parte de la institución y para los que aún no lo son , porque exponen su oferta, programas y actividades para la comunidad. Por esto todas las universidades tienen presencia en redes sociales como Facebook, Twitter y YouTube, la mayoría de estas tienen una interacción constante ya que hacen publicaciones a diario y esto genera mayor interacción con los estudiantes y personas interesadas en conocer la universidad, son pocas las que aunque tiene presencia en las redes generan contenido de manera esporádica. 


\section{REFERENCIAS}

Associaciód'Empreses i Institucions $22 \quad$ (2011). Recuperado de http://www.22network.org/index.php?idioma=esp\&id=qui_som

Cortes, Parra \& Cardona (2012). Impacto de la implementación de material digital y virtual como apoyo a la educación presencial. Evento: IV Congreso Iberoamericano de Soporte al Conocimiento con la Tecnología SOCOTE. Colombia, Bucaramanga.

D’Agostino (2007). Aspectos Teóricos de la Evaluación Educacional. San José: EUNED

De Haro, J. (2010). Redes Sociales en Educación, 1. Recuperado de: http://jjdeharo.blogspot.com/2008/11/la-redes-sociales-en-educacin.html

Fernández, A. (2004). Investigación y técnicas de mercado. Madrid: ESIC Editorial.

Goméz, H. (2003). Educación: La agenda del siglo XXI. Recuperado de http://www.ing.unal.edu.co/admfac/iei/comunicados/docs/educacion_agenda_siglo_XXI.pdf

Murcia, J. (2004). Redes del saber: investigación virtual, proceso educativo y autoformación integral. Bogotá: Colecciones Alma Mater.

Nareska, K. (2004). La investigación de mercados un enfoque aplicado. México: Editorial Pearson.

Pineda, E., Telléz, F. \& Meneses, T. (2013). Antecedentes y perspectivas Social Network Analysis and Learning Virtual Communities . Background and Perspectives Analyse des réseaux sociales et communautés virtuels d' apprentissage. Antécédents et perspectives Eliécer Pineda Ballesteros Resumen Pala. Revista Virtual Catolica Del Norte, 38(0124-5821), pp. 40-56.

Tamayo y Tamayo. (1999). La Investigación, Aprender a Investigar. Bogotá: ARFO Editores LTDA.

Vallejo, E. (2011). Enseñando a enseñar con las Tics.[En línea]. Recuperado el 10 de abril del 2013 de http://www.colombiadigital.net/aprenda-tic/formadores/como-incluir-las-tic.html

\section{SOBRE LA AUTORA}

Marcela Cardona: Magister en Gestión y Dirección de marketing global y nuevos mercados; Especialista en Gerencia en Publicidad; Profesional en Mercadeo y publicidad. En educación superior ha sido docente en Compensar Unipanamericana Institución Universitaria (2008-2014). Experiencia en la identificación de necesidades y exigencias de empresas vinculadas a diferentes sectores económicos y generación de estrategias para hacerlas competitivas en un mercado global. Docente investigadora del Grupo Investigación de Comunicación, Medios y Mercadeo. 